\title{
Guide wire displacement in robot-assisted spinal pedicle screw implantation
}

\author{
Wei Du ${ }^{1}$, Dexin Zou ${ }^{1}$, Jianfeng Zhang ${ }^{1}$, Jianqing Liu ${ }^{1}$, Wenqing $Q u^{2}$, Shudong Zhang $^{3}$ \\ ${ }^{1}$ Department of Spine, Yantaishan Hospital, Yantai, Shandong, China \\ ${ }^{2}$ Department of Traumatology, Yantaishan Hospital, Yantai, Shandong, China \\ ${ }^{3}$ Department of Joint, Yantaishan Hospital, Yantai, Shandong, China
}

Videosurgery Miniinv 2021; 16 (3): 526-535

DOI: https://doi.org/10.5114/wiitm.2021.103952

\begin{abstract}
Aim: Guide wire displacement in spinal pedicle screw implantation was analyzed in order to reduce or avoid the occurrence of this phenomenon and to reduce the complications associated with robot-assisted pedicle screw implantation surgery.

Material and methods: From April 2017 to December 2019, a retrospective study was conducted with 398 patients who underwent robot-assisted spinal pedicle screw implantation. The causes of guide wire displacement in 60 punctures were analyzed.

Results: There were 2,408 robot-assisted wire punctures of the pedicle, of which 2,348 wire punctures were located well within the pedicle, and 60 wire displacements occurred during robot-assisted wire puncture, with a displacement rate of 2.49\%. There was 1 case of thoracic segmental artery injury and 1 case of spinal cord incomplete injury. Conclusions: As it is a rare phenomenon in robot-assisted spinal pedicle screw implantation, guide wire displacement should be avoided as much as possible to improve the accuracy of screw placement and reduce surgical complications during the operation.
\end{abstract}

Key words: robot-assisted surgery, guide wire displacement, surgical complication.

\section{Introduction}

In recent years, medical robots have been widely used in spine surgery. Over 4.83 million spinal operations are performed annually around the world, with 1.34 million taking place in the United States alone [1]. Robot-assisted systems have been developed and deployed worldwide to optimize and enhance surgeon performance [2]. By adjusting movement and filtering out vibrations, the dexterity of robots has been perfected to match that of doctors' hands. The high precision and nearly unlimited endurance of robots have been praised by the industry. Literature retrieved from PubMed shows that robot-assisted surgery significant- ly improves the accuracy of screw implantation [3-6]. In a previous study, robot-assisted screw implantation that penetrated the pedicle wall $<2 \mathrm{~mm}$ accounted for approximately $95.5 \%$ [7] of all screw implants, while the accuracy of freehand screw implantation by experienced spine surgeons was approximately 92.7\% [8]. Robot-assisted screw implantation significantly improves the accuracy of this procedure, thus reducing the incidence of nerve injury and other complications. There are significant differences in the accuracy of screw placement between skilled spine surgeons and unskilled spine surgeons [9], and the use of robots has eliminated, to the greatest extent possible, the differences in screw misplacement rates due to differing 
levels of experience among physicians. However, both the human factors of surgery and the design factors of the robot itself can lead to guide wire displacement during surgical procedures, thus affecting the accuracy of the implant.

\section{Aim}

This study aimed to analyze guide wire displacement during robot-assisted spinal pedicle screw implantation to avoid guide wire displacement during surgery, to improve the accuracy of the implantation and reduce surgical complications.

\section{Material and methods}

\section{Patients}

A retrospective study was conducted with 398 patients (209 men and 189 women, aged 19 to 76 years, with an average age of 49.1 years) who underwent robot-assisted spinal pedicle screw fixation between April 2017 and December 2019. The orthopedic robot was a $3^{\text {rd }}$ generation TiRobot system (Photo 1 was downloaded from https://cn.tinavi.com/index. php? $m=$ content $\& c=$ index $\& a=$ lists $\&$ catid $=13 \#)$ produced by TINAVI Medical Technologies Co., Ltd. There were 8 cases of the cervical vertebrae, 12 cases of the upper thoracic vertebrae, 36 cases of the middle thoracic vertebrae, 134 cases of the lower thoracic vertebrae, 204 cases of the lumbar vertebrae, and 4 cases of the sacrum. Moreover, there were 6 cases of cervical vertebrae fracture, 2 cases of cervical vertebrae degeneration, 337 cases of thoracic vertebrae and lumbar vertebrae fracture, 50 cases of thoracic vertebrae and lumbar vertebrae degeneration, and 3 cases of spinal vertebrae tumor. This study was conducted in accordance with the Declaration of Helsinki. This study was conducted with approval from the Ethics Committee of Yantaishan hospital University. Written informed consent was obtained from all participants.

\section{Surgical methods}

All patients were intubated under general anesthesia, prone on a Jackson spinal table. The surgical field was disinfected, and a towel was placed.

\section{Open pedicle screw placement surgery}

After exposure of the spinous processes, laminas, and facet joints, the patient tracker is anchored on the adjacent vertebral spinous process by a clamp. The clamp connecting the patient tracker to the spinous process should be tightened and then the patient tracker is powered on. The robot is covered with a sterile cover. Then the registration is installed and placed into the operation area so that the registration is within the fluoroscopic field (Photos 2 $\mathrm{A}-\mathrm{C})$. An intraoperative computed tomography (CT) (SIEMENS ARCADIS Orbic 3D C-ARM, Germany) scan was performed, and the image was transmitted to the robotic workstation, and the pedicle screw trajectory was planned (Photo 3). This ensured the correct placement direction and specification of the pedicle screws. After the screw guider is installed, the guider is moved to the surgical field. The positioning accuracy will be displayed in the software interface in real time during the movement of the robot arm. The sleeve is placed into the screw guider. The sleeve is brought to the cortical bone surface after the bone surface is exposed. The guide wire was drilled into the vertebrae, then the optimal position was confirmed by fluoroscopy (Photos 4, 5). The cannulated tap was used to prepare the placement path first, then the screw was implanted (Photo 6).

\section{Percutaneous pedicle screw placement surgery}

A $2 \mathrm{~cm}$ skin incision was made at the cranial spinous process of the operation area, and subperiosteal stripping was performed to expose the spinous process. The patient tracker is anchored on the spinous process by a clamp. The robot is covered with

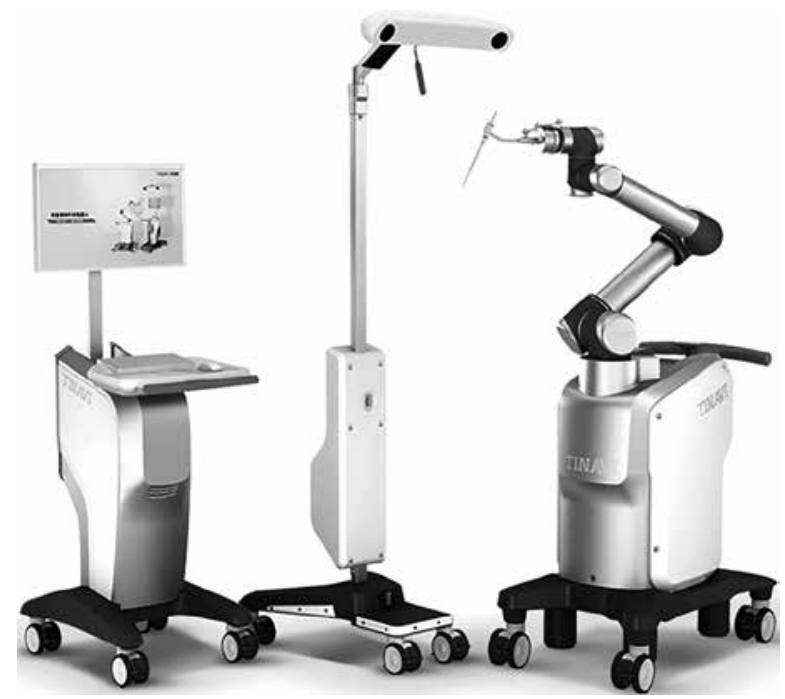

Photo 1. The China TINAVI Medical Co., Ltd. produced the $3^{\text {rd }}$ generation TiRobot system 

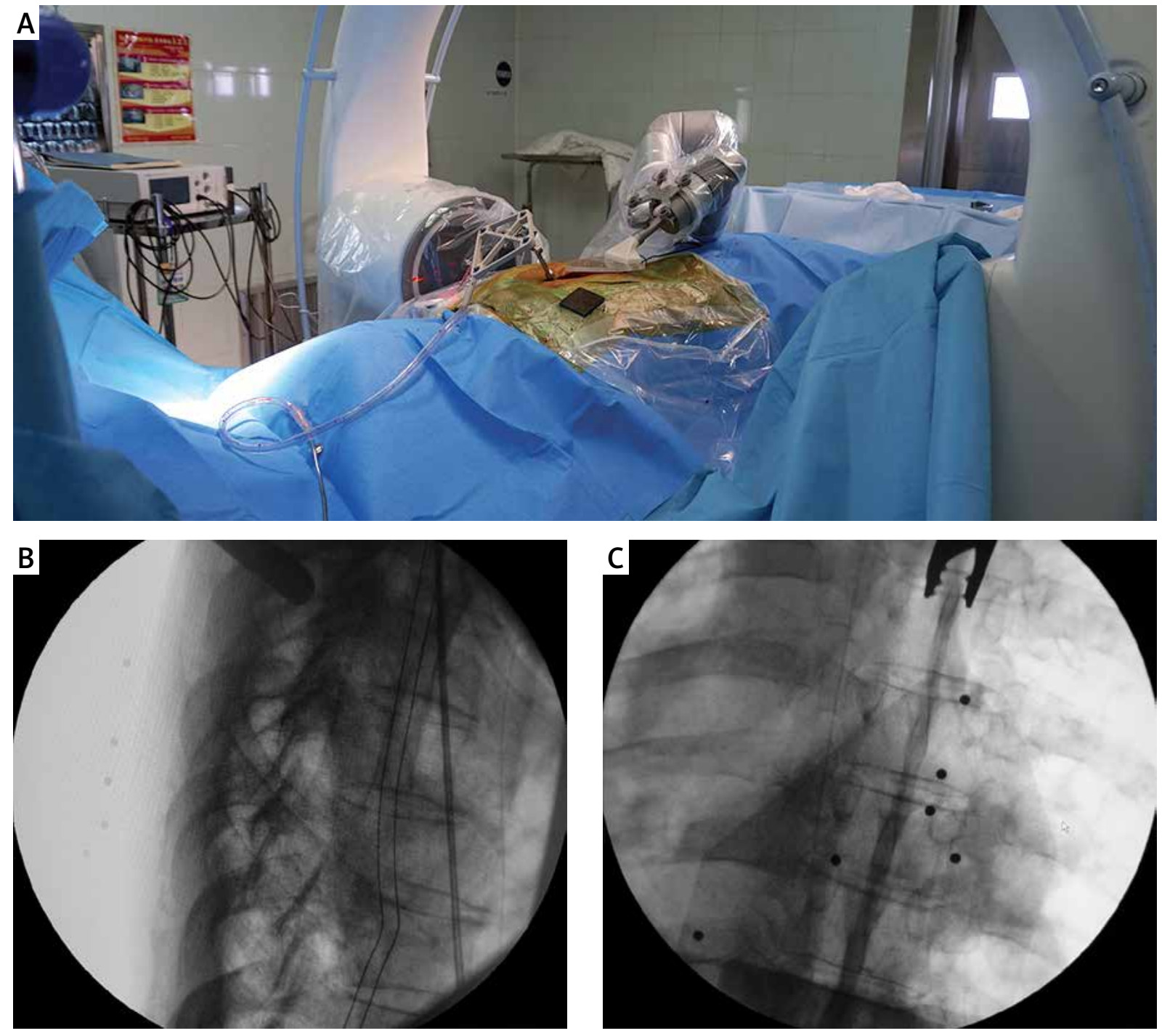

Photo 2. A - During operation a tracer and a positioning scale were installed the end of the robot and the positioning scale was placed in the operating field. B, C - X-rays show a human tracer spinous process clip that was then used to clamp the cranial spinous process of the operation area

a sterile cover. Then the registration is installed and placed into the operation area so that the registration is within the fluoroscopic field. An intraoperative CT scan was performed, and the image was transmitted to the robotic workstation. The pedicle screw trajectory was then planned, which ensured the correct placement direction and specification of the pedicle screws. After the screw guider is installed, the guider is moved to the surgical field. The positioning accuracy will be displayed in the software interface in real time during the movement of the robot arm. The sleeve is placed into the screw guider. The sleeve is brought to the cortical bone surface after the bone surface is exposed. The guide wire was drilled into the vertebrae, then the optimal position was confirmed by fluoroscopy, and a cannulated screw (UC-pass pedicle screw system) produced by Shandong Weigao Group Medical Polymer Co., Ltd was instrumented directly along the K-wire.

\section{Observation indicators}

After the completion of intraoperative robot-assisted guide wire puncture, c-arm fluoroscopy was used to observe the punctures of the thoracic and lumbar vertebrae, to observe whether the guide wire was in the pedicle, and to observe whether the guide 

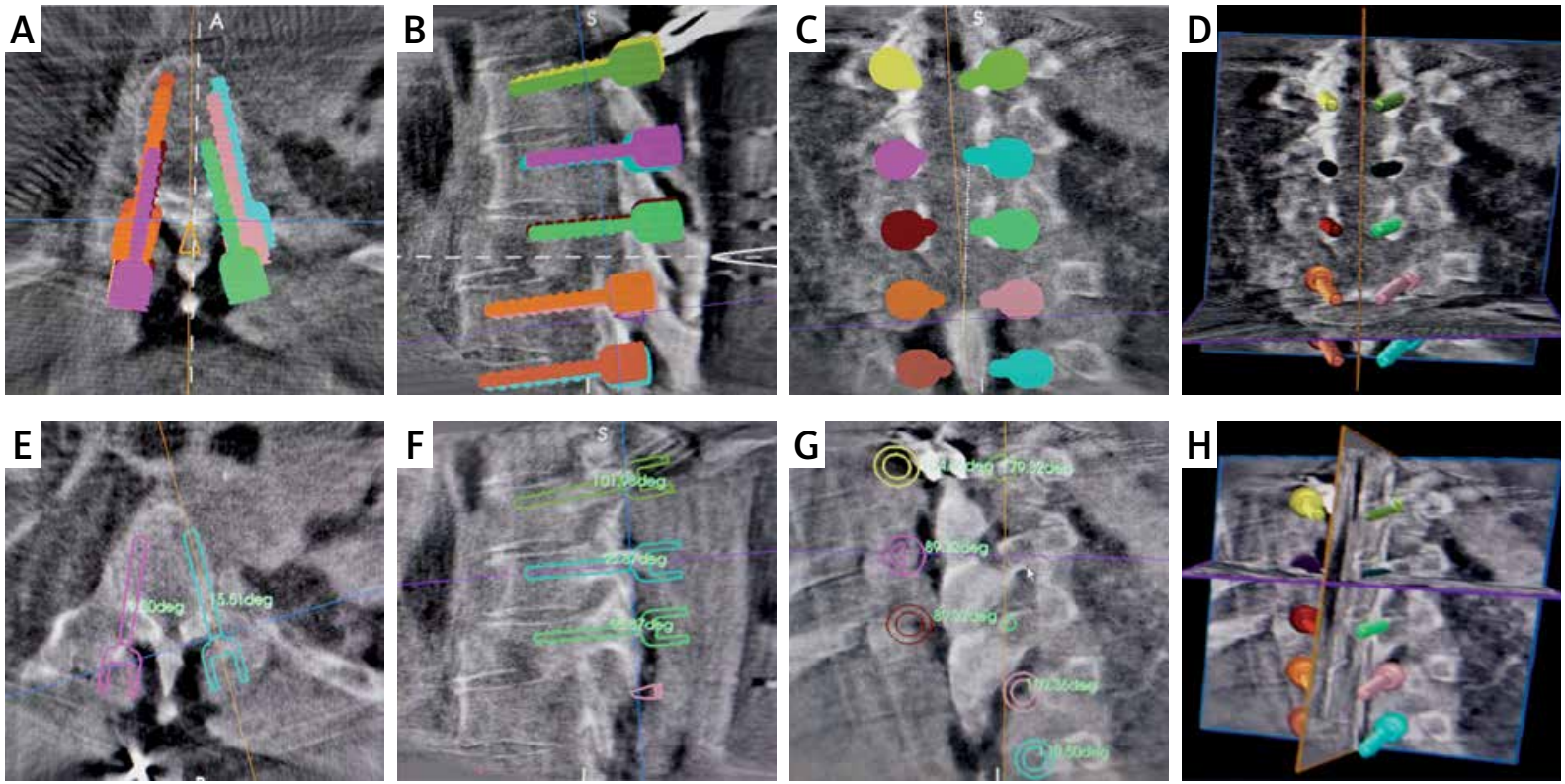

Photo 3. The pedicle screw trajectory was planned on a surgical planning and control console

wire was in the pedicle of the cervical vertebrae through the intraoperative CT scan.

\section{Results}

Robot-assisted guide wire puncture of the pedicle was performed 2,408 times, of which 2,348 were well within the pedicle, and 60 were displaced during the robot-assisted puncture, with a displacement rate of $2.49 \%$. Cervical vertebrae (C1-C7): 8 cases, displacement rate $6.25 \%$ (4/64); upper thoracic vertebrae (T1-T4): 12 cases, displacement rate $5.56 \%$ (4/72); middle thoracic vertebrae (T5-T8): 36 cases, displacement rate $0.00 \%(0 / 160)$; lower thoracic vertebrae (T9-T12): 134 cases, displacement rate $1.96 \%$ (16/816); lumbar vertebrae (L1-L5): 204 cases, displacement rate $2.81 \%$ (36/1280); sacrum $(\mathrm{S} 1,2): 4$ cases, displacement rate $0.00 \%(0 / 16)$ (Table I). Robot-assisted open pedicle screw implantation, operation time: 92-186 min, average 146.9 $\pm 48.2 \mathrm{~min}$; fluoroscopy time: $60-180 \mathrm{~s}$, average $90 \pm 28.2$ s; robot-assisted percutaneous pedicle screw implantation operation time: 49-76 min, average $62 \pm 22.9 \mathrm{~min}$; fluoroscopy time: $60-120 \mathrm{~s}$, average $69 \pm 24.2 \mathrm{~s}$; suspension of breathing during intraoperative CT localization: 60-180 s, average 80.5 $\pm 31.6 \mathrm{~s}$.

There were 34 times that the wires were displaced above the pedicle: 18 times lateral to the pedicle and

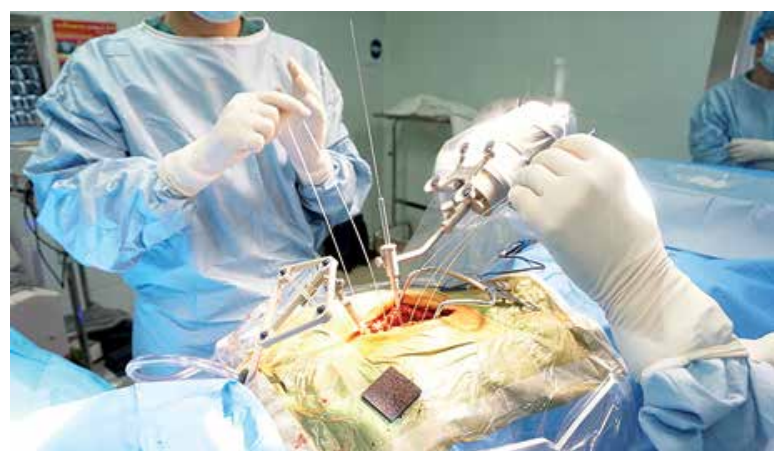

Photo 4. The guide wire was inserted along the direction of the working sleeve

8 times medial to the pedicle. The displacement of the guide wire resulted in 16 surgical bed moves. Surgical operation affects posture: 8 times; the unstable fixation of the spinal process clip: 6 times; the human tracer was far from the operating field: 2 times; the respiratory factor: 28 times. Among them, 1 case of right segmental artery injury of T11 (Photo $7 \mathrm{~A}, \mathrm{~B}$ ) resulted in massive pleural effusion on the right side (Photo $7 \mathrm{C}$ ). Arterial embolization at the right segmental artery of T11 was conducted under the guidance of intervention. A residual pleural enveloping hematoma was removed by thoracotomy 1 week after the operation. In 1 patient with cervical spondylotic disease, the left guide wires were displaced medially and punctured the walls of the pedicle from C3 to C6 (Photo 8), and the pedicle 

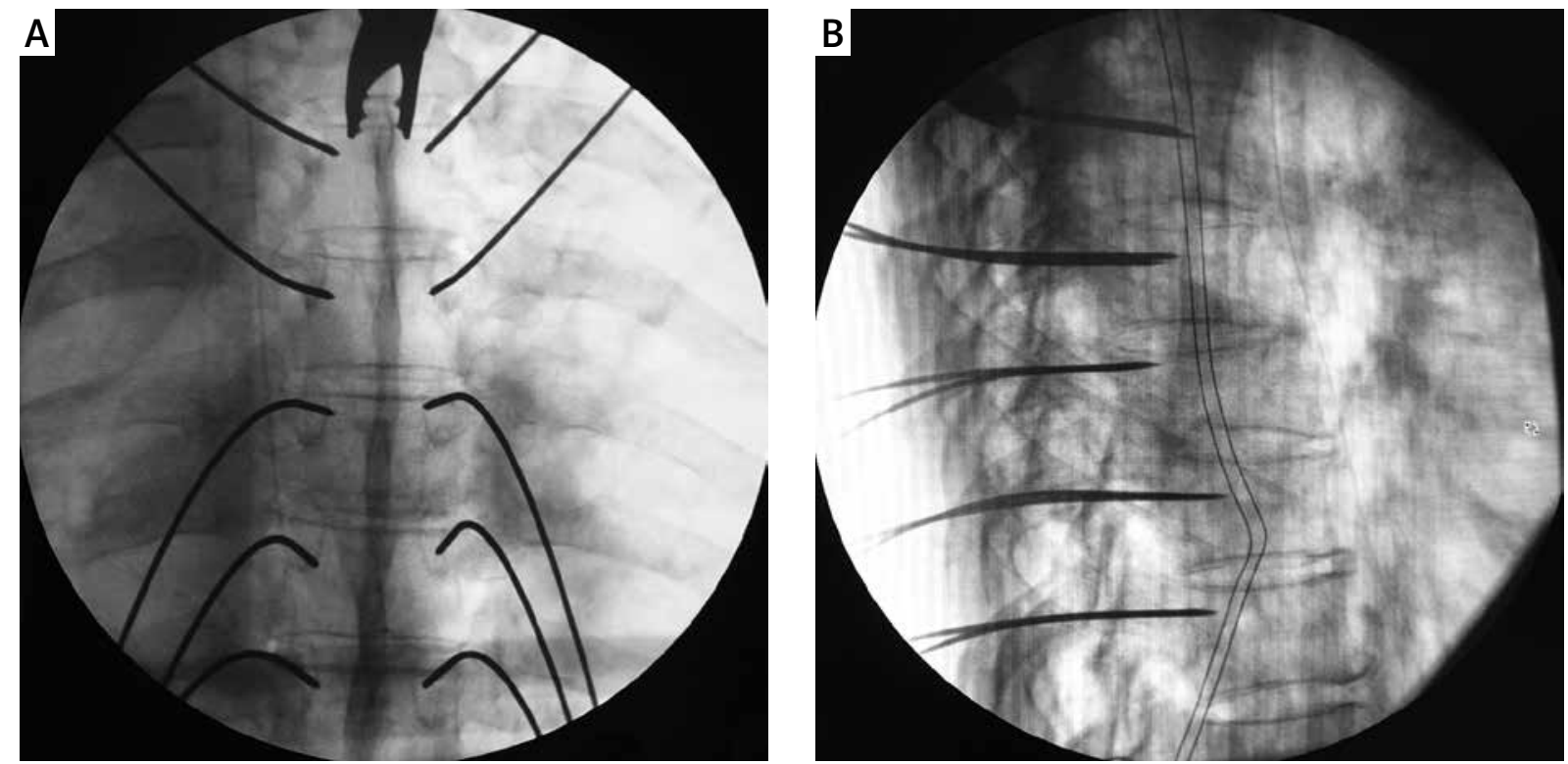

Photo 5. Intraoperative X-rays confirmed if the guide wires were in the pedicle
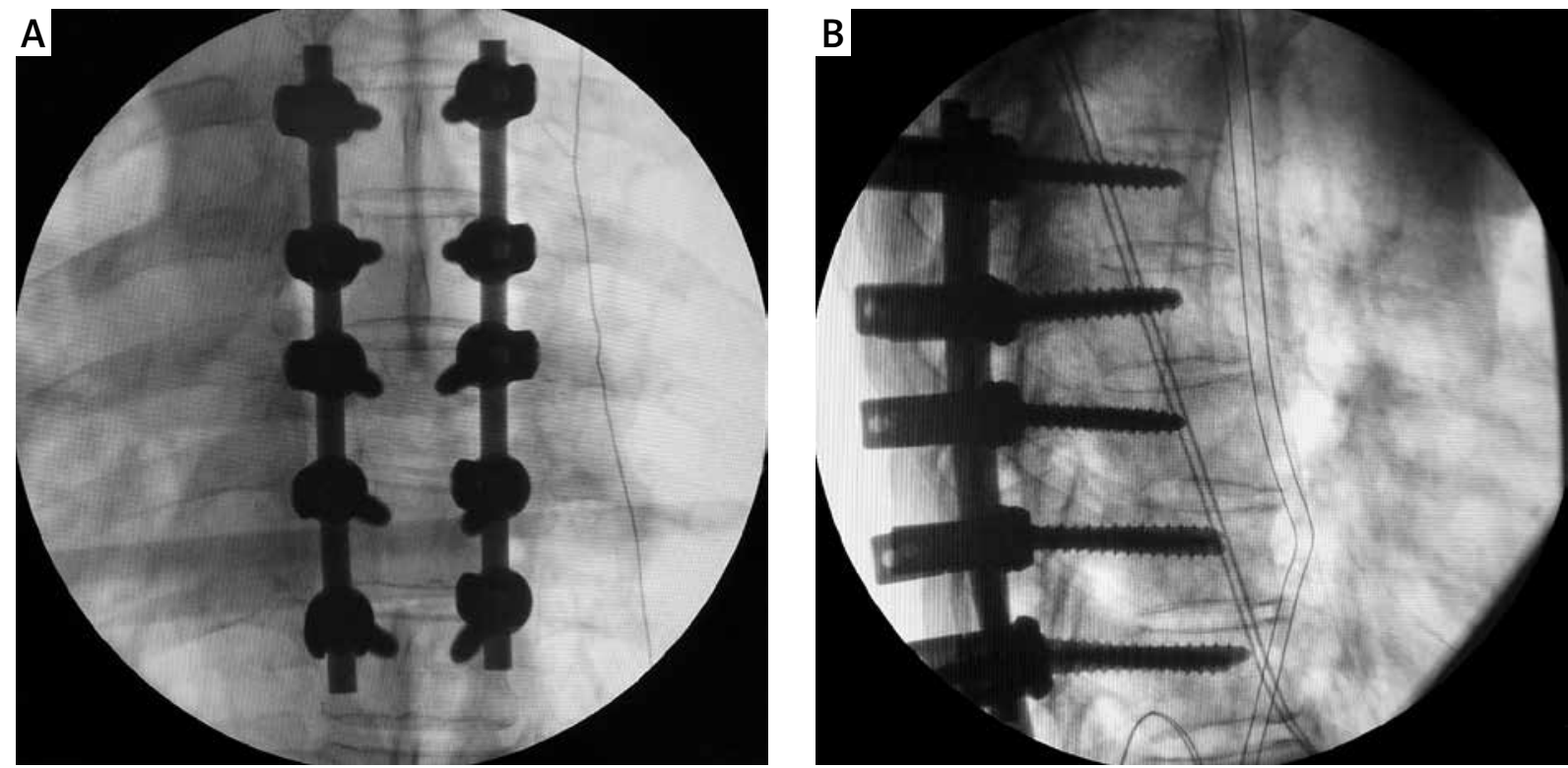

Photo 6. X-rays show the completed the fixation: A - AP fluoroscopic imaging, B - lateral fluoroscopic imaging

screw fixation was replaced by lateral mass screw fixation. The postoperative muscle strength of the patient's left upper limb was grade 3 (grade 5 before surgery), and the patient's muscle strength returned to normal after 6 months of conservative treatment. In 1 patient with thoracic vertebral fracture, the guide wire on the left side of T4 was displaced to the upper part of the pedicle, and the guide wire was removed; the pedicle screw was then implanted free- hand under c-arm guidance. For other patients with guide wire displacement, after the guide wire was pulled out intraoperatively, the insertion points and the screw trajectories were adjusted through computer re-registration and planning, and the pedicle screw was implanted accurately. Because the pedicle screw has a much larger diameter than the guide wire, it is possible for the pedicle screw to penetrate the pedicle wall, and the screw position may be 
Table I. Clinical data of the guide wire displacement in robot-assisted spinal pedicle screw implantation

\begin{tabular}{|lcccc|}
\hline Vertebrae & Number of cases & $\begin{array}{c}\text { Number } \\
\text { of punctures }\end{array}$ & $\begin{array}{c}\text { Number } \\
\text { of displacements }\end{array}$ & Displacement rate \\
\hline Cervical vertebrae (C1-C7) & 8 & 64 & 4 & $6.25 \%$ \\
\hline Upper thoracic vertebrae (T1-T4) & 12 & 72 & 4 & $5.56 \%$ \\
\hline Middle thoracic vertebrae (T5-T8) & 36 & 160 & 0 & $0 \%$ \\
\hline Lower thoracic vertebrae (T9-T12) & 134 & 816 & 16 & $1.96 \%$ \\
\hline lumbar vertebrae (L1-L5) & 204 & 1280 & 36 & $2.81 \%$ \\
\hline Sacrum (S1, 2) & 4 & 16 & 0 & $0 \%$ \\
\hline & 398 & 2408 & 60 & $2.49 \%$ \\
\hline
\end{tabular}
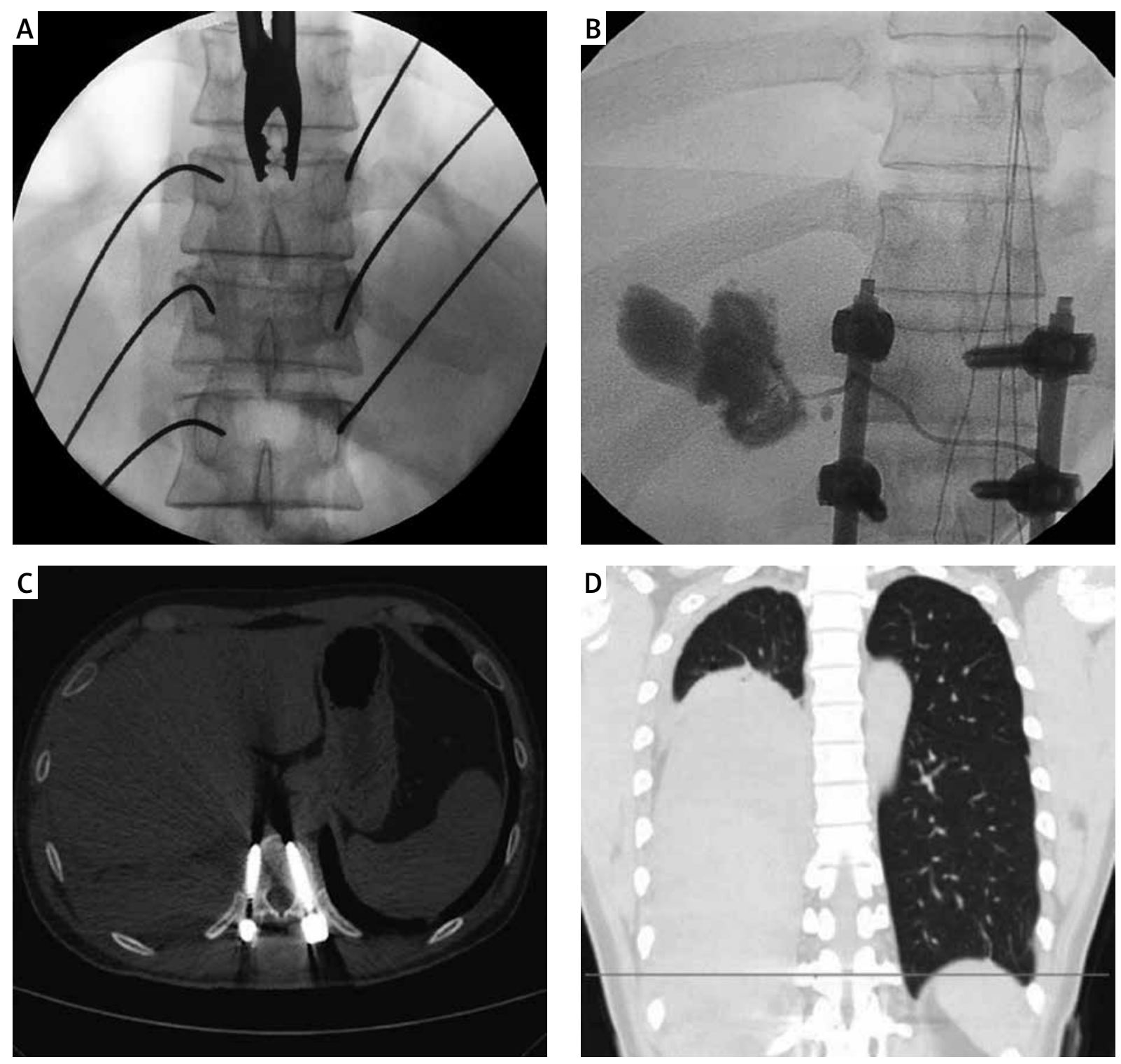

Photo 7. A - Intraoperative X-rays show that the wires were displaced laterally to the right T11 pedicle. B - Angiography shows that the right segment artery of T11 was injured. C - Postoperative CT scan shows that there was massive hemorrhage in the right thorax 

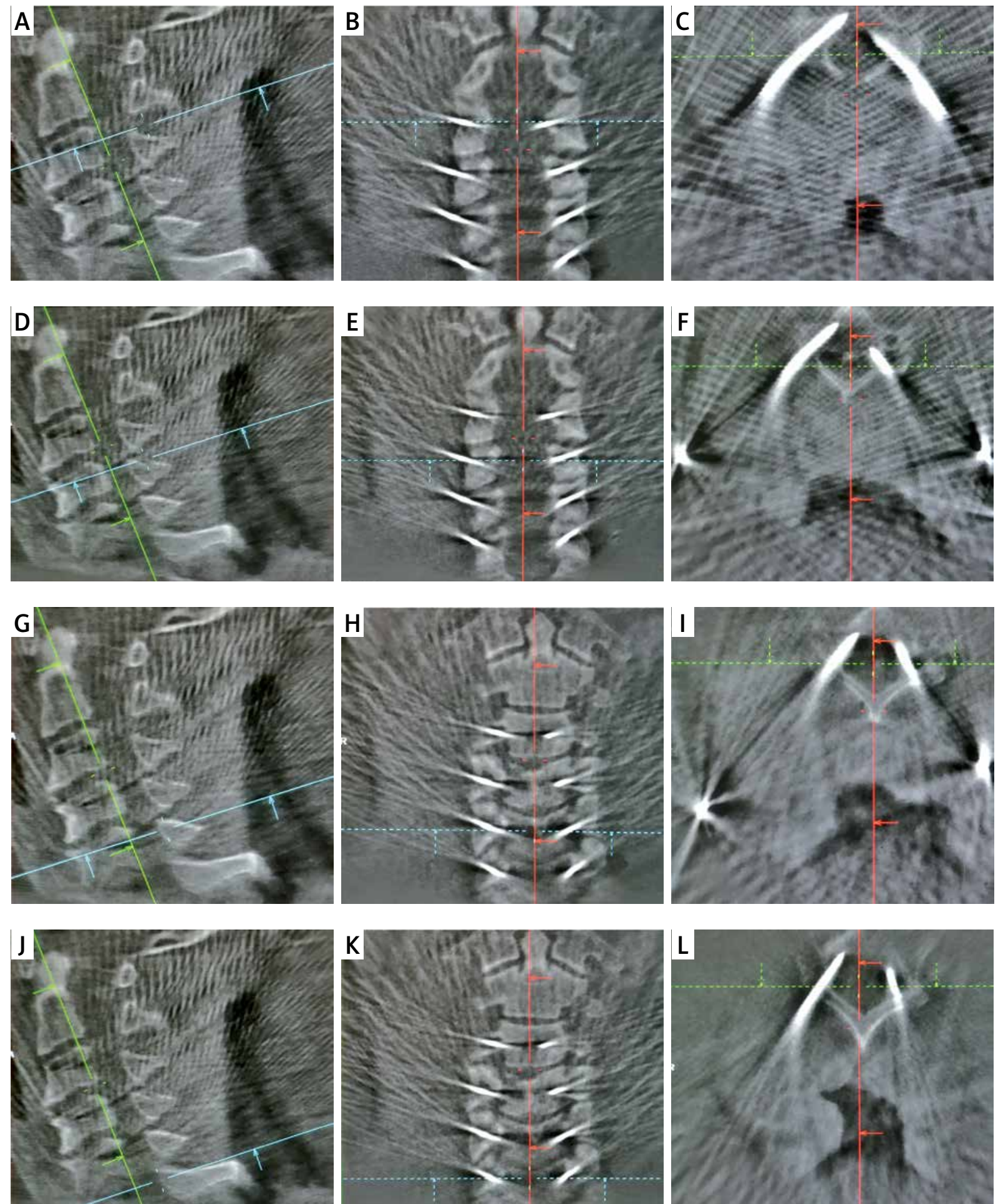

Photo 8. Intraoperative CT scans show that the left guide wires were displaced medially and through the walls of the pedicles from $\mathrm{C} 3$ to $\mathrm{C} 6$ 
changed due to human factors during screw implantation. Therefore, the pedicle screw position may be inaccurate although the guide wire is in the pedicle. Based on the Gertzbein-Robbins classification on postoperative CT scans, Grade A, screw is completely within the pedicle: $96.8 \%(2326 / 2403)$; Grade B, pedicle cortical breach < $2 \mathrm{~mm}: 3.2 \%$ (77/2403).

\section{Discussion}

Due to the lack of visual equipment, traditional orthopedic surgery relies excessively on the experience of doctors, resulting in significant surgical trauma, low accuracy, and numerous complications. Failure to implant the screws can lead to serious complications [10]. It has been reported in previous literature that the failure rate of screw implantation can reach $4.9-37.5 \%[11,12]$. In recent years, surgical robot technology has developed rapidly and has been widely studied worldwide. Surgical robot systems can overcome the physiological limitations of humans and have high operation precision, good operation repeatability, and strong operation stability $[13,14]$. These characteristics make it possible for surgical robots to be used for the internal fixation of pedicle screws and to improve the implantation accuracy. At present, there are more than 10 kinds of surgical robot systems developed for spinal surgery, including the Renaissance system (the Mazor second-generation spine Robot) [15], Da Vinci Surgical System [16], ROSA SPINE system [17], and Mazor $X$ system (the newest generation Mazor Robot) [18]. The most recognized surgical robot system is the Renaissance system, which is a spinal surgery navigation assist robot based on a parallel mechanism. The "Hover-T" technique is used to fix the robot directly to the patient's spine and guide doctors to perform spinal internal fixation. A clinical study reported that the Renaissance system had an accuracy rate of $99.4 \%$ in lumbar spine surgery [5]. However, after matching the preoperative CT scan image data with intraoperative X-ray fluoroscopy, the robot planned the pedicle screw track according to the preoperative CT data. Therefore, if the intraoperative patient's body position is inconsistent with the preoperative CT scan, the implantation accuracy might be reduced. In theory, the Renaissance system robot is less accurate than a robot with a real-time intraoperative CT scan. The TiRobot system is a new kind of tandem surgical robot system. With its assis- tance, the surgeon can plan the operation according to the intraoperative three-dimensional image. The tandem arm can cover almost all of the spine, and the real-time intraoperative navigation system makes the operation more accurate and safer. The results of previous studies have confirmed that the use of the TiRobot system can significantly improve the accuracy of pedicle screw implantation and does not result in any postoperative complications; in one study, the mean deviation for each screw was 1.5 $\pm 0.8 \mathrm{~mm}$ [19], meeting the accuracy requirements of screw internal fixation of the upper cervical spine [20]. However, the TiRobot system, Renaissance robot system, and other robot systems can be located through the navigation principle [21], leading to the displacement of the guide wire and errors, and leading to a decrease in the accuracy of the implant. Therefore, through a retrospective study, this paper analyzed guide wire displacement in spine surgery using the TiRobot and strived to improve the accuracy and safety of the implant and reduce surgical complications. Guide wire displacement occurs when the guide wire penetrates the pedicle cortex and not the pedicle. The guide wire may be displaced above, below, medially, or laterally to the pedicle.

\section{Principle of guide wire displacement with the TiRobot}

Through intraoperative three-dimensional CT scanning, the TiRobot can construct a three-dimensional virtual human body that is $1: 1$ with the size of the physical body and can ensure that the spatial position is exactly matched to the human body. By detecting the relative position of the robot tracer and the human body tracer through the navigation system, it can guide the robot to find the 3D virtual human body planning screw trajectory. Therefore, if the physical body and the 3D virtual human body do not match, displacement will occur.

\section{Causes and treatment of guide wire displacement}

Reasons for guide wire displacement may include the following: 1 . The fixation of the surgical bed is not reliable during the operation, and the surgical bed moves. 2. The soft tissue is pulled during the operation, resulting in a change of body position. 3. The surgical operation affects posture. 4. Respiratory factors: Breathing can make the patient's 
body position change with the degree of respiratory movement, which may cause a body position shift; vertebral movement caused by respiratory movement: after intubation under general anesthesia, the respiratory movement caused vertebral movement, among which T7 $(2.26 \mathrm{~mm})$, T12 (2.27 mm), and L4 $(1.61 \mathrm{~mm})$ had the greatest influence. Vertebral movement caused by respiratory movement has a greater influence on the head-tail direction [22]. Due to the movement of joints, such as the intervertebral discs in the spine, respiratory mobility will cause different spinal joints to have varying degrees of motion, thus causing the relative displacement of human trackers and physical bodies.

Solution: 1. The surgical bed: The components are reliably fixed, and the operation can reduce the microtremor of the surgical bed as much as possible; 2. Soft tissue pulling, resulting in resistance when placing the locating sleeve: when cutting the skin and deep fascia, try to follow the direction set by the mechanical arm to reduce resistance to the locating sleeve; 3. During open surgery: soft tissue exposure does not affect the positioning of the robot cannula; 4. Stop the ventilator during positioning: stop the ventilator when oxygen saturation is $>95 \%$.

After the human tracer was fixed to the human body, the robot camera regarded the human tracker as a local marker of the human body, and the camera located the 3D virtual human body after comprehensive calculation based on the human tracer and intraoperative CT scan. Therefore, the displacement of the human tracer and the physical human body will cause the 3D virtual human body implant to change, resulting in displacement. Reasons for the relative displacement include the following: 1 . The human tracker is far from the operation area, and the camera is based on the human tracer and intraoperative $\mathrm{CT}$ scan. After comprehensive calculation, the positioning error of the 3D virtual human body is larger. 2. The spinous process clamp is not firmly fixed.

Solution: 1. The human tracker should be as close to the surgical area as possible without affecting the operation. 2. The spinous process clamp should be fixed firmly, and the operation should be gentle, being careful to avoid touching. Once the spinous process clamp is touched and displaced, and after the clamp is firmly fixed, the operation should be stopped immediately and repeated from the beginning.

Other causes: The insertion point is too close to the steep bone surface and slips while placing the locating sleeve. Therefore, in the preoperative design, try not to select the nailing point on the relatively steep bone surface, and increase the "holding force" between the guide wire and the bone surface. Before inserting the guide wire, the guide sleeve is used to grind a shallow hollow at the insertion point to reduce the slip of the guide wire.

\section{Intraoperative notes}

Intraoperative precautions: improve safety awareness, recalculate, and refer to the clinical experience and bone structure; if something is in doubt, fluoroscopy will assist the surgeons' judgment. Try to avoid pulling the patient. The guide wire puncture operation is gentle and steady.

Guide wire displacement is a rare phenomenon in robot-assisted spinal pedicle screw implantation. During the operation, guide wire displacement should be avoided as much as possible, to improve the accuracy of the screw implantation and reduce surgical complications.

\section{Conclusions}

Guide wire displacement is a rare phenomenon in robot-assisted spinal pedicle screw implantation. It should be avoided as much as possible to improve the accuracy of screw placement and reduce surgical complications during the operation.

\section{Acknowledgments}

The National Key Research and Development Program of the Ministry of Science and Technology of the People's Republic of China (2017YFC0110600); the Science and Technology Program of Yantai City (2019YD033).

\section{Conflict of interest}

The authors declare no conflict of interest.

\section{References}

1. Open PR. Global Surgical Robots for the Spine Industry Trend, Growth, Shares, Strategy and Forecasts 2016 to 2022; 2017. Available from: https://www.openpr.com/news/442943/global-surgical-robots-for-the-spine-industry-trend-growth-sharesstrategy-and-forecasts-2016-to-2022.html. Accessed October 25, 2019.

2. D'Souza M, Gendreau J, Feng A, et al. Robotic-assisted spine surgery: history, efficacy, cost, and future trends. Robot Surg 2019; 6: 9-23. 
3. Kantelhardt SR, Martinez R, Baerwinkel S, et al. Perioperative course and accuracy of screw positioning in conventional, open robotic-guided and percutaneous robotic-guided, pedicle screw placement. Eur Spine J 2011; 20: 860-8.

4. Li HM, Zhang RJ, Shen CL. Accuracy of pedicle screw placement and clinical outcomes of robot-assisted technique versus conventional freehand technique in spine surgery from nine randomized controlled trials: a meta-analysis. Spine 2020; 45: E111-9.

5. Kim HJ, Jung WI, Chang BS, et al. A prospective, randomized, controlled trial of robot-assisted vs freehand pedicle screw fixation in spine surgery. Int J Med Robot 2017; 13. doi: 10.1002/ rcs.1779.

6. Wang JQ, Wang Y, Feng Y, et al. Percutaneous sacroiliac screw placement: a prospective randomized comparison of robot-assisted navigation procedures with a conventional technique. Chin Med I 2017; 130: 2527-34.

7. Le X, Tian W, Shi Z, et al. Robot-assisted versus fluoroscopy-assisted cortical bone trajectory screw instrumentation in lumbar spinal surgery: a matched-cohort comparison. World Neurosurg 2018; 120: e745-51.

8. Marcus HJ, Cundy TP, Nandi D, et al. Robot-assisted and fluoroscopy-guided pedicle screw placement: a systematic review. Eur Spine J 2014; 23: 291-7.

9. Lee CH, Hyun SJ, Kim YJ, et al. Accuracy of free hand pedicle screw installation in the thoracic and lumbar spine by a young surgeon: an analysis of the first consecutive 306 screws using computed tomography. Asian Spine J 2014; 8: 237-43.

10. Inamasu J, Guiot BH. Vascular injury and complication in neurosurgical spine surgery. Acta Neurochir 2006; 148: 375-87.

11. Tian NF, Huang QS, Zhou P, et al. Pedicle screw insertion accuracy with different assisted methods:a systematic review and meta-analysis of comparative studies. Eur Spine J 2011; 20: 846-59.

12. Devito DP, Kaplan L, Dietl R, et al. Clinical acceptance and accuracy assessment of spinal implants guided with Spine Assist surgical robot: retrospective study. Spine 2010; 35: 2109-15.

13. Bertelsen A, Melo J, Sánchez E, et al. A review of surgical robots for spinal interventions. Int J Med Robot 2013; 9: 407-22.

14. Kostrzewski S, Duff JM, Baur C, et al. Robotic system for cervical spine surgery. Int J Med Robot 2012; 8: 184-90.

15. Dreval' ON, Rynkov IP, Kasparova KA, et al. Results of using Spine Assist Mazor in surgical treatment of spine disorders. Zh Vopr Neirokhir Im N N Burdenko 2014; 78: 14-20.

16. Lee JYK, Bhowmick DA, Eun DD, et al. Minimally invasive, robot-assisted, anterior lumbar interbody fusion: a technical note. J Neurol Surg A Cent Eur Neurosurg 2013; 74: 258-61.

17. Lonjon N, Chan-Seng E, Costalat V, et al. Robot-assisted spine surgery: feasibility study through a prospective case-matched analysis. Eur Spine J 2016; 25: 947-55.

18. Khan A, Meyers JE, Siasios I, et al. Next-generation robotic spine surgery: first report on feasibility, safety, and learning curve. Oper Neurosurg 2019; 17: 61-9.

19. Han X, Tian W, Liu Y, et al. Safety and accuracy of robot-assisted versus fluoroscopy-assisted pedicle screw insertion in thoracolumbar spinal surgery: a prospective randomized con- trolled trial. J Neurosurg Spine 2019; 1-8. doi: 10.3171/2018.10. SPINE18487.

20. Tian W. Robot-assisted posterior c1-2 transarticular screw fixation for atlantoaxial instability: a case report. Spine 2016; 41 Suppl 19: B2-5.

21. Overley SC, Cho SK, Mehta Al, et al. Navigation and robotics in spinal surgery: where are we now? Neurosurgery 2017; 80: S86-99.

22. Liu Y, Zeng C, Fan M, et al. Assessment of respiration-induced vertebral motion in prone-positioned patients during general anaesthesia. Int J Med Robot 2016; 12: 214-8.

Received: 11.09.2020, accepted: 19.11.2020. 